\title{
Comparison of Lumboperitoneal Shunt and Stereotactic Ventriculoperitoneal Shunt in the Treatment of Refractory Idiopathic Intracranial Hypertension: A Case Report and Literature Review
}

\author{
Changsong $\mathrm{Gao}^{1,2}$, Hongtao Zhang ${ }^{2}$, and Jidi Fu' ${ }^{3, *}$ \\ ${ }^{1}$ Clinical Medical College of Weifang Medical University, Shandong, China \\ ${ }^{2}$ Department of Neurosurgery, Yantai Yuhuangding Hospital Affiliated to Qingdao University, Shandong, China \\ ${ }^{3}$ Plastic Surgery Hospital, Chinese Academy of Medical Sciences and Peking Union Medical College, Beijing, China
}

*Corresponding authors: Jidi Fu, Plastic Surgery Hospital, Chinese Academy of Medical Sciences and Peking Union Medical College, 33 Badachu Road, Shijingshan District, Beijing, 100043,China, E-mail: fujidi2006@126.com

Received: 19 May, 2021 | Accepted: 23 Jun, 2021 | Published: 30 Jun, 2021

Citation: Gao C, Zhang H, Fu J (2021) Comparison of Lumboperitoneal Shunt and Stereotactic Ventriculoperitoneal Shunt in the Treatment of Refractory Idiopathic Intracranial Hypertension: A Case Report and Literature Review. J Clin Case Stu 6(2): dx.doi.org/10.16966/24714925.224

Copyright: (C) 2021 Gao C, et al. This is an open-access article distributed under the terms of the Creative Commons Attribution License, which permits unrestricted use, distribution, and reproduction in any medium, provided the original author and source are credited.

\begin{abstract}
Idiopathic Intracranial Hypertension (IIH) or Pseudotumor Cerebri (PTC) is a disorder of young obese females and characterized by headache, papilledema and raised intracranial pressure. However, it is in the absence of known pathological cause. Due to the uncertainty of etiology, it may lead to misdiagnosis and grave clinical prognosis. IIH is typically treated with Lumboperitoneal Shunting (LPS) and Ventriculoperitoneal Shunting (VPS), but shunts are prone to dysfunctions and infection, resulting in recurrent headaches in many patients after this treatment. We report a case of 41-year-old obese female (BMI: 30.9 ) with IIH, who has a history of hypertension (Blood pressure: $150 / 100 \mathrm{mmHg}$ ) and elevated intracranial pressure (Open pressure: $450 \mathrm{mmH}_{2} \mathrm{O}$ ). After the failure of several medical treatments, the patient was offered LPS because of vision loss and headache, but the postoperative symptoms (intermittent headache, mainly total craniocerebral prickling pain with neck and shoulder pain) were not significantly relieved for 11 years. Therefore, considering the blockage of the primary shunt tube and the small ventricle in the patient, it has difficulty in puncture ventricle puncture. We have to treat with the stereotactic VPS (SVPS) for her exacerbation symptoms. More surprisingly, the hypertension was in the normal range $(<115 / 80 \mathrm{mmHg}$ ) after the surgery (without taking antihypertensive medication). To compare the surgical therapeutic effects and complications of LPS and the SVPS in the treatment of idiopathic intracranial hypertension. Cerebrospinal Fluid (CSF) diversion with VPS or LPS is usually performed when the main symptom is vision loss; it also stabilizes headache and papilledema. LPS significantly alleviates symptoms in the short term, but due to excessive shunt of LPS for a long time, it is easy to be complicated with Chiari malformation and slit ventricle syndrome. Therefore, we encourage operating the SVPS on our patients for the favorable long-term outcome.
\end{abstract}

Keywords: Idiopathic intracranial hypertension; Lumboperitoneal shunt; The stereotactic ventriculoperitoneal shunt; Case report

Abbreviations: IIH: Idiopathic Intracranial Hypertension; LPS: Lumbarperitoneal Shunt; VPS: Ventriculoperitoneal Shunt; SVPS: Stereotactic Ventriculoperitoneal Shunt; CSF: Cerebrospinal Fluid; CT: Computer Tomography; MRI: Magnetic Resonance Imaging

\section{Introduction}

Theoretical and Research Basis for Treatment Idiopathic intracranial hypertension (IIH) is a disease characterized by elevated intracranial pressure, which can lead to severe headache, papilledema, and vision loss without an identifiable cause. The incidence of IIH is 1 to 3 per 100,000 people per year, which predominantly affects young adult females under the age of 45 [1]. The initial treatments of options for IIH are conservative, including weight loss and conservative treatment. Advanced cases of severe vision loss and/or progressive IIH may not be effective for conservative treatment. Such advanced cases occur in $10 \%$ to $20 \%$ of patients with IIH. Patients may progress to permanent blindness through optic nerve atrophy at highly varying rates (weeks to years) if left untreated. Therefore, surgery is recommended for advanced IIH cases [2]. The common surgical treatments of IIH are LPS and VPS [3]. While the ventricles are not enlarged, the LPS are usually preferred over the VPS [4]. However, due to long-term excessive shunt of LPS, Chiari malformation and slit ventricle syndrome are easy to occur. In addition, the SVPS was noted to have a higher medium-term patency rate than LPS [5]. Therefore, we compared the surgical therapeutic effects and complications of LPS with the SVPS for the treatment of refractory IIH.

\section{Case Presentation}

Eleven years ago, a 41-year-old obese female (BMI 30.9) who had a 7-year history of hypertension (BP: $150 / 100 \mathrm{mmHg}$ ) and elevated intracranial pressure (OP: $450 \mathrm{mmH}_{2} \mathrm{O}$ ) with IIH. After the failure of 
several medical treatments, the patient was send to the neurosurgery department for intermittent binocular vision loss and amaurosis lasting for one year. The condition was further aggravated and accompanied by intermittent headache. On admission, the patient was fully conscious, and the hemodynamics was stable. After examination, the visual acuity was $90 / 100$ in her right eye and 20/100 in the left. Neurological examination showed bilateral abducent nerve paralysis, severe optic papilla edema (grade 4) and unclear boundaries in the fundus of the optic never (Figure 1).

Considering the possibility of an intracranial mass lesion, cranial magnetic resonance imaging (MRI) was performed and no structural changes were found (Figures $2 \mathrm{~A}$ and $2 \mathrm{~B}$ ). Brain MRI plain scan and CSF analysis were normal. Repeated lumbar puncture showed pressure $>330 \mathrm{mmH}_{2} \mathrm{O}$. No abnormalities were found in the other examination cranial nerves. These results are consistent with idiopathic intracranial hypertension.

The patient was intending to surgery and underwent LPS on the $18^{\text {th }}$ day of referral. The postoperative vision was improved and the optic papilla edema was reduced. She was diagnosed with idiopathic intracranial hypertension and discharged. During the follow-up 2 years, her binocular was significantly improved, the visual acuity was 100/100 in her right eye and 100/100 in the left. The optic nerve papillary edema was less than before (Figure 3).

However, during the 11 years after LPS treatment, the weight of patient was no significant change (BMI 29.7) and the blood pressure was maintained at $150 / 100 \mathrm{mmHg}$. Therefore, the patient was admitted to the hospital several times for intermittent headache, mainly the whole craniocerebral prickling pain accompanied by neck and shoulder pain. Patient was no significant relieved with painkillers, and CSF pressure was measured by multiple lumbar puncture. The overall pressure showed an upward trend, and the final pressure was $350 \mathrm{mmH}_{2} \mathrm{O}$. Fortunately, there was no significant change in the patient's vision after the first operation. According to the lumbar puncture pressure of the patient, we considered that the primary shunt blockage caused by the small ventricle (Figure 4A) leads to some difficulties in ventriculocentesis. MRI brain T1 images show Chiari malformation (Figure 4B). Therefore, we decided to perform VPS in stereotaxic manner (preoperative BP: 150/95 mmHg; BMI 29.7). She experienced instant relief (the headache was significantly relieved) after the surgery and documented intracranial pressure (OP: $140 \mathrm{mmH}_{2} \mathrm{O}$ ). Computer tomography (CT) of the brain is shown after surgery (Figures 5A and 5B). More surprisingly, the patient's blood pressure was within the normal range $(<115 / 80 \mathrm{mmHg})$ after several days of measurement (without taking antihypertensive medications). Finally, she was diagnosed with refractory IIH and discharged with hospital. During the follow-up 8 months, the patient's blood pressure remained within the normal range $(120 / 80 \mathrm{mmHg})$ (without taking antihypertensive medications) and visual acuity was improved further. More importantly, the patient had no symptoms of headache or nausea during follow-up to date. Reexamination of the head MRI image showed the Chiari malformation was slightly relieved (Figure 5C). We can be sure that the SVPS worked better than the LPS.

\section{Discussion}

IIH is a rare disease with elevated intracranial pressure of unknown etiology [6]. The incidence of IIH is 1 to 3 per 100,000 people per year [1], which mainly affects obese females. About $90 \%$ of patients diagnosed with IIH are obese females [6]. It is unclear why obesity is so closely related to the development of IIH. However, it is considered that the increase of intra-abdominal pressure can lead to an increase in right ventricular filling pressure, resulting in an increase in venous pressure [7]. Three typical symptoms of a typical IIH include severe daily headaches, papilledema, and vision loss [8]. Papilledema is generally considered to be the primary symptom of IIH and can lead to severe morbidity if left untreated [9]. The $10 \%$ to $20 \%$ of IIH patients have advanced cases and may progress to permanent blindness through
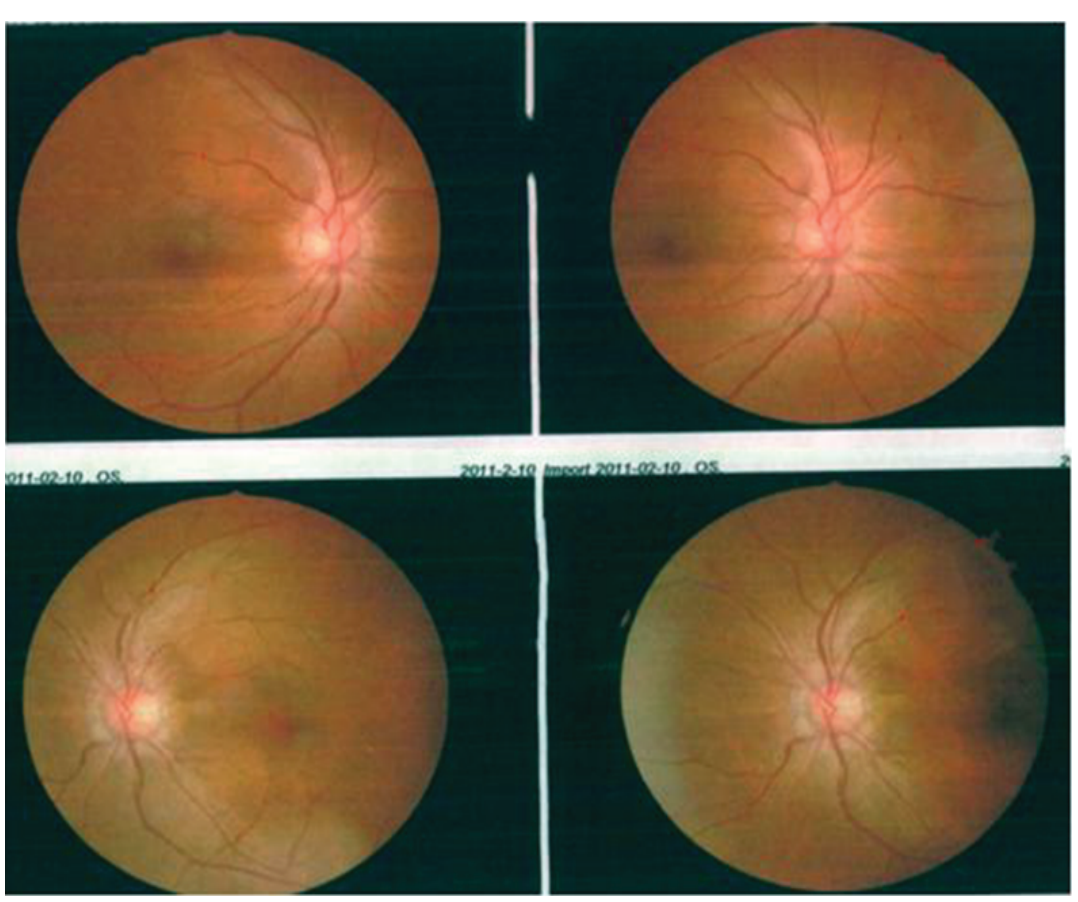

Figure 1: Bilateral optic fundi showed papilledema.

Citation: Gao C, Zhang H, Fu J (2021) Comparison of Lumboperitoneal Shunt and Stereotactic Ventriculoperitoneal Shunt in the Treatment of Refractory Idiopathic Intracranial Hypertension: A Case Report and Literature Review. J Clin Case Stu 6(2): dx.doi.org/10.16966/2471- 


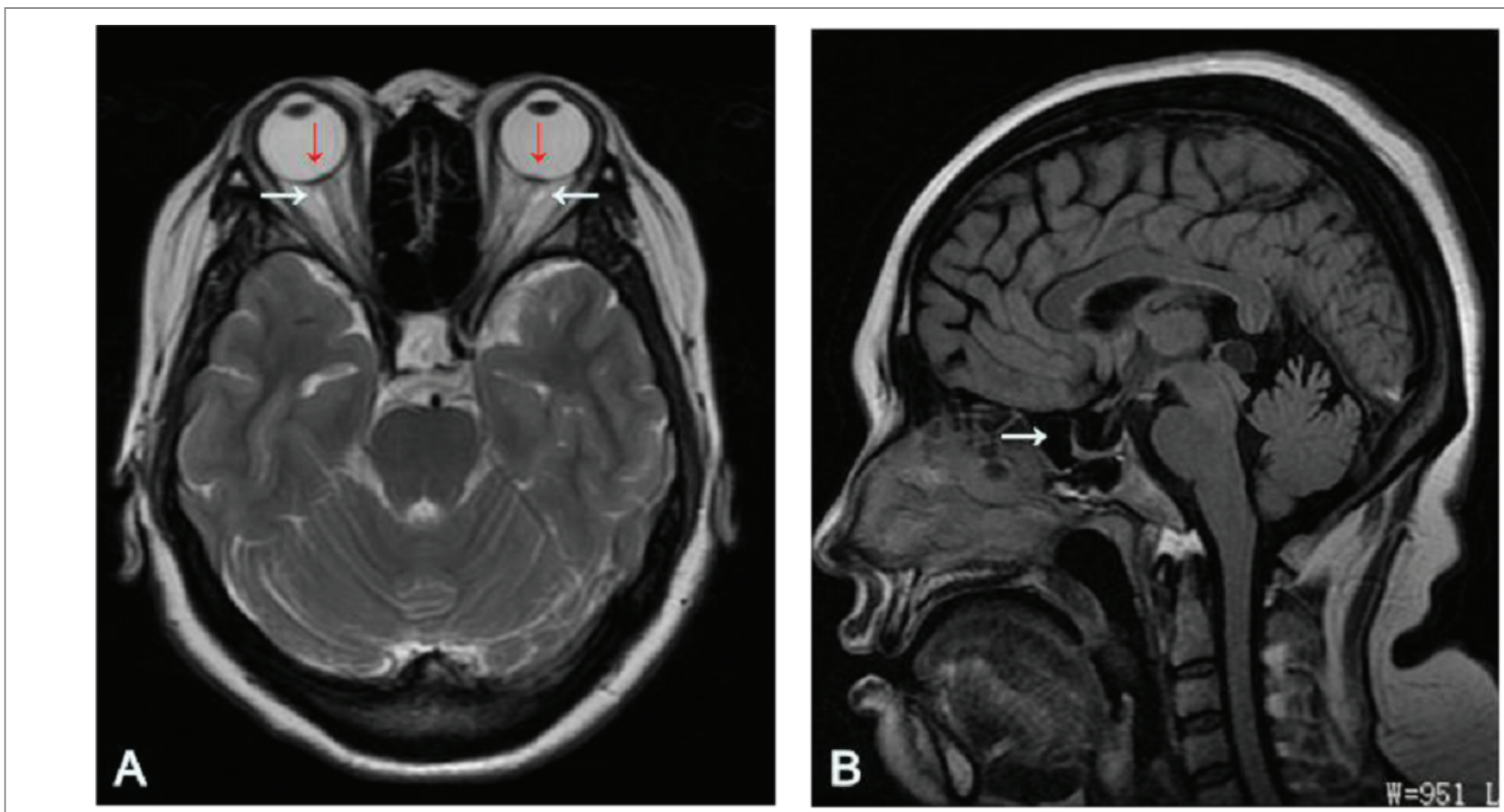

Figure 2: A) Axial T2 weighted image showing increased transverse dimension of bilateral optic nerve/sheath complexes (white arrows) and bilateral optic nerve papillae protrusion into the vitreous space of the globes with flattening of the bilateral posterior sclera (red arrows). B) Sagittal T1 image showing empty sella (white arrow).
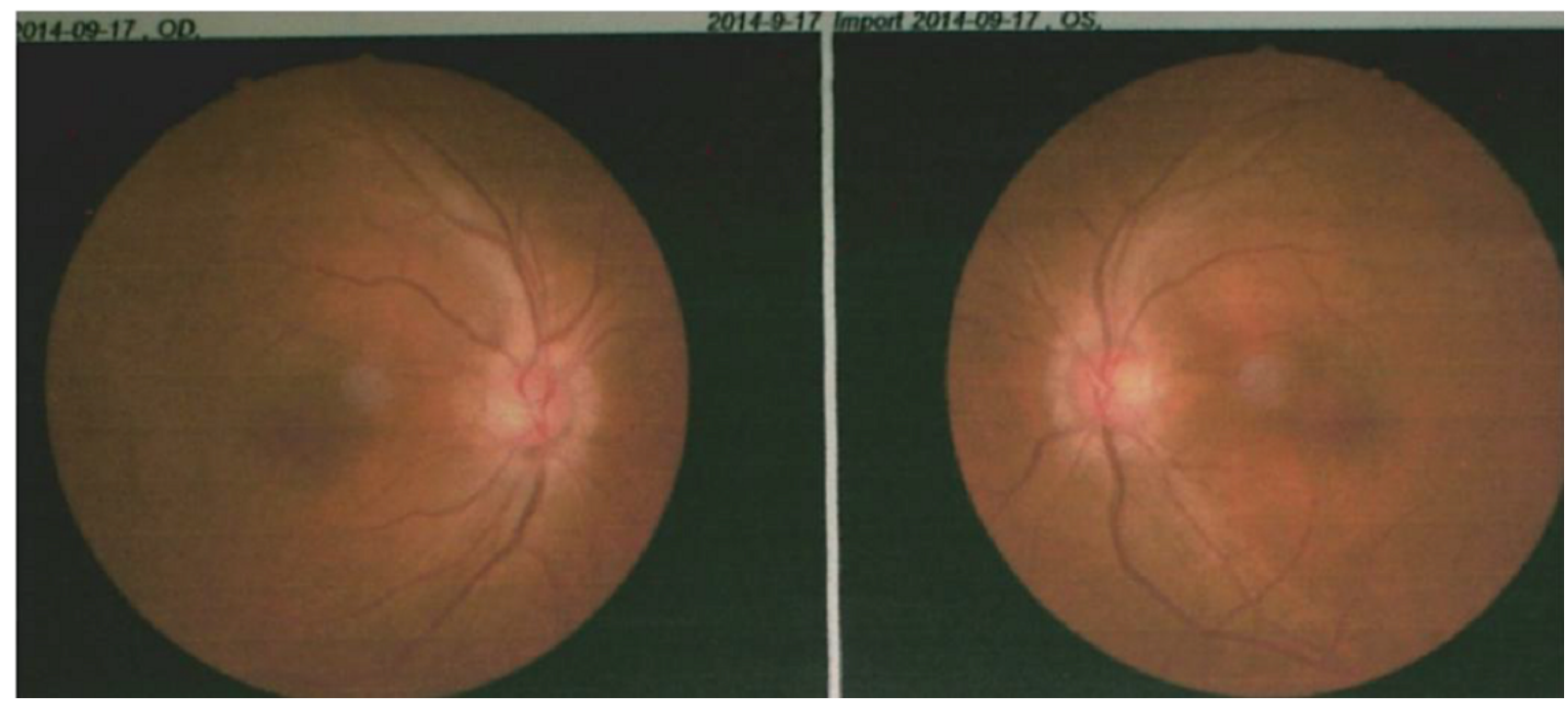

Figure 3: The optic papilla edema was reduced.

optic nerve atrophy at the rate of high changes in (weeks to years) [5]. Although headache is the most frequently reported symptom, loss of vision is remains the most worrying complication [10].

The exact cause of IIH is not clear, but some mechanisms have been proposed to explain its occurrence, such as CSF hyper secretion, normal CSF access blocked, CSF absorption disorder, venous sinus thrombosis and some 137 unknown causes. The predominately diagnosis of IIH is exclusion [11]. Table 1 for the modified Dandy diagnostic criteria.
For patients with simple vision loss, drug therapy may be effective in the short term, but long-term use may lead to gradual vision loss and even the risk of blindness. Therefore, patients with ineffective conservative treatment should be treated by operation. Dandy [12] believes that increased cerebral blood flow or cerebrospinal fluid volume leads to increased intracranial pressure, leading to symptoms related to intracranial hypertension and papillary edema, leading to progressive vision loss and blindness. Therefore, IIH is usually treated with shunts. Both LPS and VPS can reduce the compression of the 


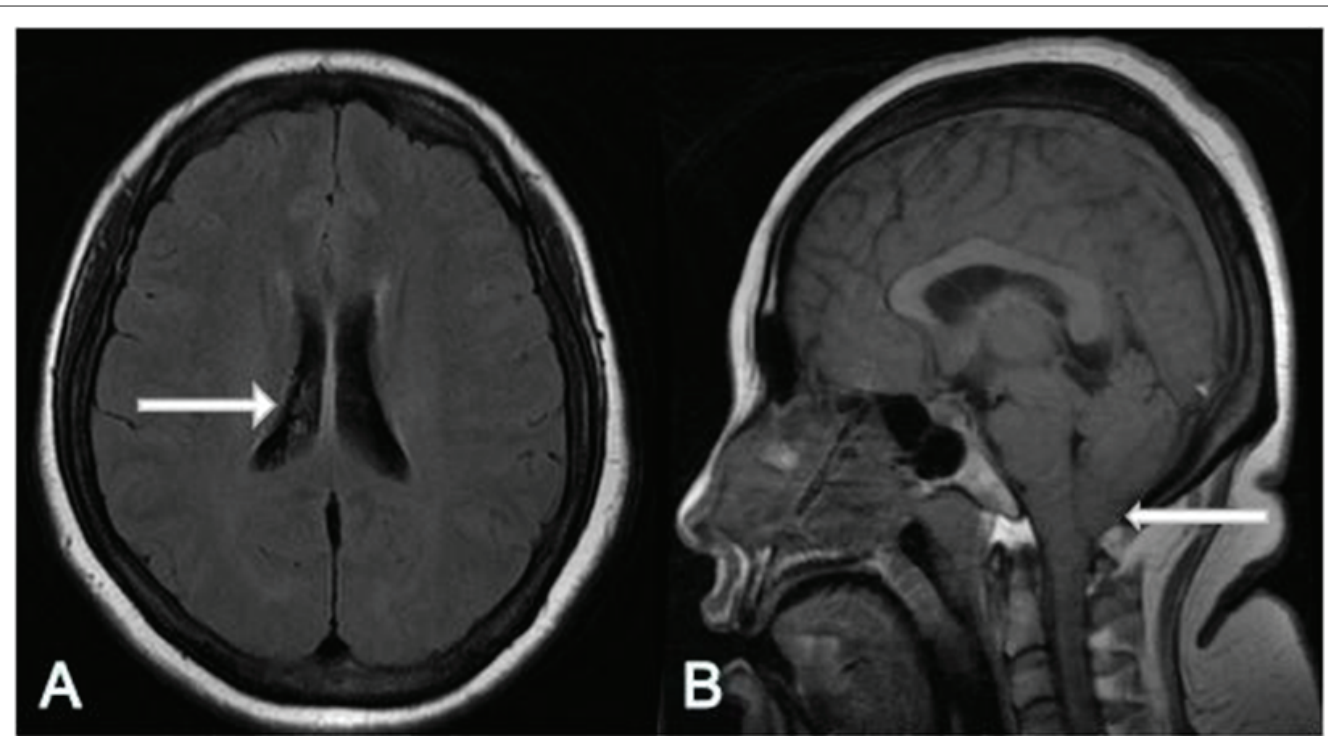

Figure 4: A) Axial T1 image showing normal volume of lateral ventricle (white arrow). B) Sagittal T1 image showing Chiari malformation (white arrow).
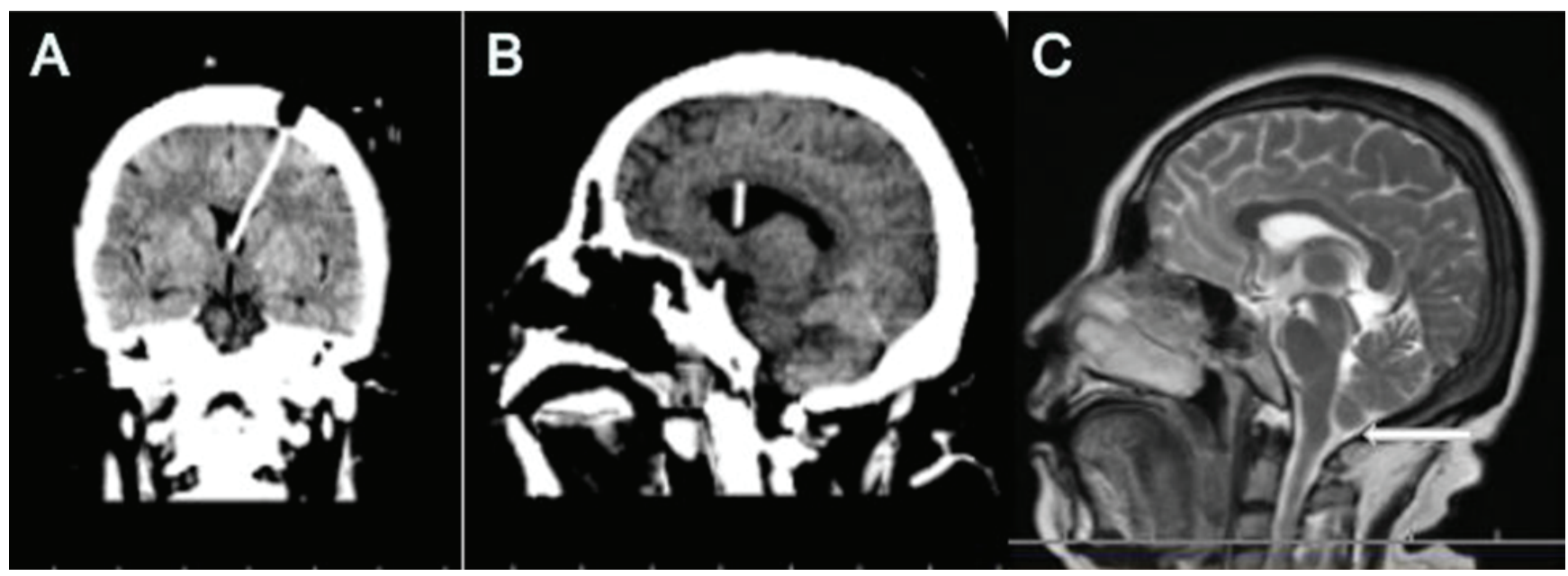

Figure 5: A) Coronal CT image showing the ventricular shunt tube was accurately located in the ventricle; B) Sagittal CT image showing the ventricular shunt tube after the stereotactic VPS; C) Sagittal T2 image showing the Chiari malformation was slightly relieved (white arrow).

Table 1: Modified Dandy Criteria for the diagnosis of IIH.

\section{Modified Dandy Criteria}

Signs and symptoms of increased ICP

No localizing neurologic finding (except possible $6^{\text {th }}$ nerve palsy)

Normal CT/MRI findings

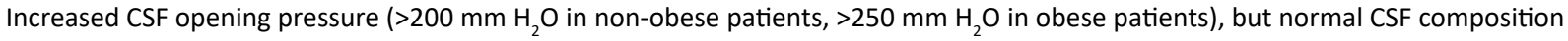

No other identifiable cause of increased ICP

Citation: Gao C, Zhang H, Fu J (2021) Comparison of Lumboperitoneal Shunt and Stereotactic Ventriculoperitoneal Shunt in the Treatment of Refractory Idiopathic Intracranial Hypertension: A Case Report and Literature Review. J Clin Case Stu 6(2): dx.doi.org/10.16966/2471- 
arteriovenous around the optic nerve and reduce the intracranial pressure by shunting cerebrospinal fluid, which has a certain effect on visual acuity. LP and VP shunts are safe and effective in the treatment of headache and papilledema caused by IIH, and the improvement of visual acuity loss and/or visual field defect. The short-term effect is good. The main complications of LPS include reduction intracranial pressure caused by excessive shunt, blockage of shunt tube, infection, and cerebrospinal fluid leakage [13]. In addition, due to excessive LPS, it is easy to be complicated with Chiari malformation and slit ventricle syndrome. In addition, several reports show that the revision rates of LPS technology for Chiari malformation [14] and radicular pain are $60 \%, 4 \%$ and $18.5 \%[3,15]$. Therefore, in order to avoid the formation of Chiari malformation and slit ventricle syndrome, the postoperative CSF pressure of LPS was higher than VPS, so VPS has better effect on headache and vision recovery in patients with idiopathic intracranial hypertension than LPS. In addition, $18 \%$ to $85 \%$ of cases reported complications after LPS treatment [16]. Due to the need for multiple revision surgeries and frequent wound complications, many patients tend to abandon this treatment and in favor of VPS for IIH. However, shunt tube blockage is a common complication of VPS. The obstruction of the end of the ventricle is mainly due to excessive shunt of CSF, the smaller ventricle, and the end of the ventricle is buried in the brain tissue. It is possible that shunt failure rates may be similar between LP and VP shunts, but reasons for revision, removal, or replacement may differ. Therefore, it is necessary to find a solution to solve the blockage of the shunt tube-the SVPS technology. Nowadays, with the rise of the global obesity rate, IIH tends to become more and more common [17]. At present, CSF shunting remains the primary treatment to reduce the severity of acute symptoms. The effectiveness and safety of VPS of IIH has been greatly improved with the emergence of image-guided technology. Image guidance can be used to perform surgery safely and accurately. Some series use image guidance, and each patient only needs one catheter to place VPS $100 \%$ [18]. And the mediumterm opening rate of SVPS is significantly higher than that of LPS [5]. Consequently, it might reduce the rate of shunt revision, particularly those due to proximal obstructions [19]. More surprisingly, the associated symptoms/triggers of IIH have also been addressed, such as hypertension. Therefore, we need to further study the relationship between hypertension and IIH in hypertensive IIH patients.

\section{Conclusion}

When the main symptoms are visual loss, stable headache and papilledema, VPS or LPS is usually performed for CSF shunt. LPS significantly alleviates symptoms in the short term, but long-term excessive shunt is easy to be complicated with Chiari malformation [14] and slit ventricle syndrome. In addition, we believe that SVPS technology is the first option for our patients. Because compared with more invasive surgical procedures, it is relatively simple, minimally invasive, ease to control morbidities, and long-term effect. Therefore, we encourage our patients to apply the SVPS for the favorable longterm outcome.

\section{Declaration of Conflicting Interests}

The author(s) declared no potential conflicts of interest with respect to the research, authorship, and/or publication of this article.

\section{Funding}

The author(s) received no financial support for the research, authorship, and/or publication of this article.

\section{Note}

Informed written consent was obtained for treatment and research purposes. Patients were assured that any data presented from their treatment would be anonymous, and thus these cases have been deidentified and some details of the cases have been changed to protect confidentiality.

\section{References}

1. Wakerley BR, Tan MH, Ting EY (2015) Idiopathic intracranial hypertension. Cephalalgia 35: 248-261.

2. Aoki N (1990) Lumboperitoneal shunt: clinical applications, complications, and comparison with ventriculoperitoneal shunt. Neurosurgery 26: 998-1003; discussion 1003-1004.

3. Burgett RA, Purvin VA, Kawasaki A (1997) Lumboperitoneal shunting for pseudotumor cerebri. Neurology 49: 734-739.

4. Johnston I, Besser M, Morgan MK (1988) Cerebrospinal fluid diversion in the treatment of benign intracranial hypertension. J Neurosurg 69: 195-202.

5. Hollenbach E, Acheson J, Wadley J, Palmer J (2000) Visual results in stereotactic ventriculo-peritoneal (VP) shunts-a new treatment for idiopathic intracranial hypertension (IIH). Neuro-ophthalmology 23: 193.

6. Boyter E (2019) Idiopathic intracranial hypertension. JAAPA. 32: 3035.

7. Rekate HL (2007) Hydrocephalus and idiopathic intracranial hypertension. J Neurosurg 107: 435-437; discussion 437-438.

8. Burkett JG, Ailani J (2018) An Up to Date Review of Pseudotumor Cerebri Syndrome. Curr Neurol Neurosci Rep 18: 33.

9. Daggubati LC, Liu KC (2019) Intracranial Venous Sinus Stenting: A Review of Idiopathic Intracranial Hypertension and Expanding Indications. Cureus 11: e4008.

10. McGeeney BE, Friedman DI (2014) Pseudotumor cerebri pathophysiology. Headache 54: 445-458.

11. Thurtell MJ, Wall M (2013) Idiopathic intracranial hypertension (pseudotumor cerebri): recognition, treatment, and ongoing management. Curr Treat Options Neurol 15: 1-12.

12. Dandy WE (1937) Intracranial Pressure Without Brain Tumor: Diagnosis and Treatment. Ann Surg 106: 492-513.

13. Satti SR, Leishangthem L, Chaudry MI (2015) Meta-Analysis of CSF Diversion Procedures and Dural Venous Sinus Stenting in the Setting of Medically Refractory Idiopathic Intracranial Hypertension. AJNR Am J Neuroradiol 36: 1899-904.

14. Chumas PD, Armstrong DC, Drake JM, Kulkarni AV, Hoffman HJ, et al. (1993) Tonsillar herniation: the rule rather than the exception after lumboperitoneal shunting in the pediatric 233 population. J Neurosurg 78: 568-73.

15. Eggenberger ER, Miller NR, Vitale S (1996) Lumboperitoneal shunt for the treatment of pseudotumor cerebri. Neurology 46: 15241530.

16. Karabatsou K, Quigley G, Buxton N, Foy P, Mallucci C (2004) Lumboperitoneal shunts: are the complications acceptable? Acta Neurochir (Wien) 146: 1193-1197.

17. Markey KA, Mollan SP, Jensen RH, Sinclair AJ (2016) Understanding idiopathic intracranial hypertension: mechanisms, management, and future directions. Lancet Neurol 15: 78-91. 
18. Woodworth GF, McGirt MJ, Elfert P, Sciubba DM, Rigamonti D (2005) Frameless stereotactic ventricular shunt placement for idiopathic intracranial hypertension. Stereotact Funct Neurosurg 83: 12-16.

19. Abu-Serieh B, Ghassempour K, Duprez T, Raftopoulos C (2007) Stereotactic ventriculoperitoneal shunting for refractory idiopathic intracranial hypertension. Neurosurgery 60: 1039-1043; discussion 1043-1044. 